\title{
MiR-455-3p inhibits the degenerate process of chondrogenic differentiation through modification of DNA methylation
}

\author{
Hao Sun ${ }^{1}$, Xiaoyi Zhao ${ }^{1}$, Chengyun Zhang ${ }^{1}$, Ziji Zhang ${ }^{1}$, Jiayong Lun ${ }^{1}$, Weiming Liao ${ }^{1}$ and Zhiqi Zhang ${ }^{1}$
}

\begin{abstract}
The aim of this work was to determine whether miR-455-3p regulates DNA methylation during chondrogenic differentiation of hMSCs. The expression of miR-455-3p and de novo methyltransferase DNMT3A was assessed in micromass culture of hBMSCs, which induced chondrogenic differentiation in vitro, and in E16.5 mice in vivo. A luciferase reporter assay was used to confirm whether miR-455-3p directly targets DNMT3A by interaction with the 3'UTR. Using an Illumina Infinium Methylation EPIC microarray, genome-wide DNA methylation of hBMSCs with or without overexpressed miR-455-3p was examined for 28 days during induced chondrogenic differentiation. Here, we showed that miR-455-3p was more expressed during the middle stage of hBMSC chondrogenic differentiation, and less expressed in the late stage. DNMT3A was less expressed in the middle stage and more expressed in the late stage, and was also more expressed in the palms of miR-455-3p deletion mice compared to those of wild-type mice. The luciferase reporter assay demonstrated that miR-455-3p directly targets DNMT3A 3'-UTR. miR-455-3p overexpression inhibits the degenerate process during chondrogenic differentiation, while deletion of miR-455-3p in mice accelerated cartilage degeneration. Genome-wide DNA methylation analysis showed miR-455-3p overexpression regulates DNA methylation of cartilage-specific genes. GO analysis revealed PI3K-Akt signaling pathway was most hypomethylated. Our data show that miR-455-3p can regulate hMSC chondrogenic differentiation by affecting DNA methylation. Overexpression of miR-455-3p and DNA methylation inhibitors can thus potentially be utilized to optimize chondrogenic differentiation.
\end{abstract}

\section{Introduction}

It is widely accepted that DNA methylation occurs at the sites of CpG dinucleotides, plays a critical role in the regulation of gene expression, and is involved in a variety of biological processes ${ }^{1,2}$. DNA methylation is regulated by DNA methyltransferases (DNMTs), which promote methylation, and by the ten-eleven translocation (TET) family of $5 \mathrm{mC}$ dioxygenases, which are involved in demethylation. There are three mainly active DNMT

Correspondence: Weiming Liao (liaowmsysu@163.com) or

Zhiqi Zhang (zhzhiqi@mail.sysu.edu.cn)

${ }^{1}$ Department of Joint Surgery, First Affiliated Hospital of Sun Yat-Sen University,

510080 Guangzhou, Guangdong, China

These authors contributed equally: Hao Sun, Xiaoyi Zhao

Edited by G. Calin enzymes, namely $D N M T 1, D N M T 3 A$, and $D N M T 3 B^{3}$; $D N M T 1$ is the major maintenance methyltransferase while $D N M T 3 A$ and $D N M T 3 B$ are primarily de novo methyltransferases ${ }^{4}$.

The effect of DNA methylation in osteoarthritis (OA) pathophysiology is becoming increasingly evident. OA progress is accompanied by methylation changes to cartilage catabolic and developmentally associated genes such as MMP13, SOX9, ADAMTS4 ${ }^{5-9}$. DNA has been shown to be differentially methylated between normal and OA cartilage ${ }^{5}$. For example, the enhancer region of NF- $\mathrm{KB}$ at $-5.8 \mathrm{~kb}$ was significantly demethylated in OA cartilage compared with the same region in control samples, leading to the activation of inducible nitric oxide synthase

\section{(c) The Author(s) 2018}

(c) (i) Open Access This article is licensed under a Creative Commons Attribution 4.0 International License, which permits use, sharing, adaptation, distribution and reproduction c. in any medium or format, as long as you give appropriate credit to the original author(s) and the source, provide a link to the Creative Commons license, and indicate if changes were made. The images or other third party material in this article are included in the article's Creative Commons license, unless indicated otherwise in a credit line to the material. If material is not included in the article's Creative Commons license and your intended use is not permitted by statutory regulation or exceeds the permitted use, you will need to obtain permission directly from the copyright holder. To view a copy of this license, visit http://creativecommons.org/licenses/by/4.0/. 
to start or accelerate OA process ${ }^{6}$. Recent studies showed that $M M P 13^{7}$ and $A D A M T S 4^{8}$ decreased methylation and $S O X 9^{9}$ increased methylation in $\mathrm{OA}$, and the gene expression is inversely related to methylation. Moreover, increased DNA methylation due to age and oxidative stress impaired the differentiation capacity of hMSCs ${ }^{10}$, and DNA methyltransferase inhibitors promoted the differentiation of stem cells by repressing DNA methylation $^{11}$.

MicroRNAs (miRNAs) are short ( 22 nt), non-coding, single-stranded RNAs that function as post-transcriptional regulators. Specifically, miRNAs silence gene expression by binding to the $3^{\prime}$-untranslated region (3'-UTR) of specific target mRNAs, thereby promoting their degradation or inhibiting their translation ${ }^{12}$. Previously, we found high expression levels of miR-455-3p in human adipose-derived stem cells (hADSCs) during chondrogenic differentiation ${ }^{13}$. We subsequently demonstrated that miR-455-3p plays a role in regulating $\mathrm{OA}$ and the chondrogenic differentiation of hMSCs ${ }^{14-16}$.

Recent studies have shown that miR-455-3p regulates certain pathological processes, such as cancer ${ }^{17}$. Our previous study showed that miR-455-3p functions as an activator for early chondrogenic differentiation of ATDC5 cells, and directly targets and inhibits the expression of Runt-related transcription factor 2 (RUNX2) ${ }^{15}$. We also demonstrated that miR-455-3p promotes chondrogenic differentiation by suppressing the expression of $H D A C 2$ and $H D A C 8$, thereby maintaining an appropriate level of histone $\mathrm{H} 3$ acetylation at the COL2A1 promoter to promote the production of type II collagen ${ }^{14}$. However, whether miR-455-3p regulates cartilage development and degeneration by adjusting DNA methylation level is not clear. Using miRNA target prediction algorithms, we found that miR-455-3p has a potential target in $D N M T 3 A$, which does regulate DNA methylation. Thus, we hypothesized that miR-455-3p plays an important role in hMSC chondrogenic differentiation by regulating the ability of DNMT3A to modulate DNA methylation. In this study, we investigated the role of miR-455-3p in the process of hMSC chondrogenic differentiation by characterizing its effect on DNA methylation of cartilagespecific genes and pathways.

\section{Results}

\section{Expression pattern of miR-455-3p and DNMT3A during chondrogenic differentiation of hBMSCs}

To characterize the expression patterns of miR-455-3p and DNMT3A during chondrogenic differentiation, hBMSCs were induced to differentiate into chondrocytes in micromass culture in vitro. Safranin O staining at days 21 and 35 showed successful chondrogenic differentiation of hBMSCs (Supplementary Figure 1). The expression of miR-455-3p increased rapidly in hBMSC chondrogenic differentiation beginning on day 7 , peaked at 21 days, and was followed by a marked decrease from days 28 to 35 (Fig. 1a). Meanwhile, the opposite trend was evident in DNMT3Aexpression, which descended from days 3 to 21 and then rapidly increased from days 28 to 35 (Fig. 1b). Immunohistochemistry analysis of DNMT3A (Fig. 1c) also showed a similar trend to RT-PCR, suggesting that miR-455-3p may affect the expression of DNMT3A.

Our previous study ${ }^{14}$ revealed that while moderate and high levels of miR-455-3p expression were detected in proliferating and prehypertrophic chondrocytes, little to no miR-455-3p expression was observed in hypertrophic chondrocytes in vivo by in situ hybridization in E16.5 mouse limbs. Therefore, we performed DNMT3A staining in E16.5 mouse limbs to characterize the expression patterns of DNMT3A. The result showed while low levels of DNMT3A expression were detected in proliferating chondrocytes, moderate and high levels of DNMT3A expression was observed in prehypertrophic and hypertrophic chondrocytes (Fig. 1d, e). These contrary expression patterns between miR-455-3p and DNMT3A indicated the co-regulation of chondrogenesis.

\section{MiR-455-3p regulates the expression of DNMT3A in vitro and in vivo}

To further investigate whether miR-455-3p regulates $D N M T 3 A$ expression in vitro and in vivo, we inhibited and overexpressed miR-455-3p. The chondrocytes isolated from normal cartilage were transfected with either miR-455-3p or anti-miR-455-3p (Fig. 2a, c). The expression levels of DNMT3A and SOX9 were assessed by qRT-PCR and western blotting. Treatment with miR-455-3p resulted in significantly decreased mRNA expression of DNMT3A and an increase in SOX9 expression (Fig. 2b, e, f). Meanwhile, inhibition of miR-455-3p resulted in a significant increase in the expression of DNMT3A and a decrease in SOX9 expression (Fig. 2d-f).

We constructed miR-455-3p deletion mice and proved the successful deletion. We compared miR-455-3p expression between 6-month wild-type mice and homozygous mice by in situ hybridization. The results showed miR-455-3p were significantly decreased on cartilage (Fig. 2g-i), bone (Supplementary Figure 2a, b, e), and meniscus (Supplementary Figure 2c, d, e). For the in vivo study, the embryos with wild-type, heterozygous, and homozygous genotypes were obtained through the mating of heterozygous male and female mice. We isolated the forelimbs of mice at embryonic stage 16.5 (E16.5) and characterized the expression patterns of DNMT3A. Notably, we observed high DNMT3A expression levels in palms of miR-455-3p deletion mice (Fig. 2l, m), while low levels were detected in wild-type mice (Fig. 2j, k). These results suggest that miR-455-3p may affect the expression of DNMT3A and regulate cartilage metabolism. 
$\mathrm{a}$

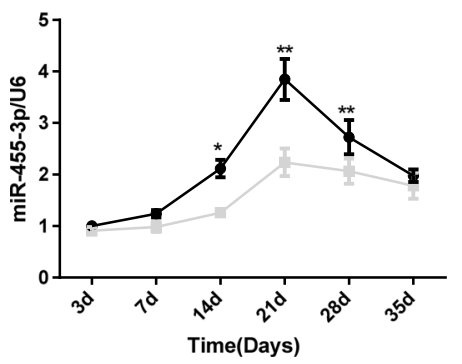

b

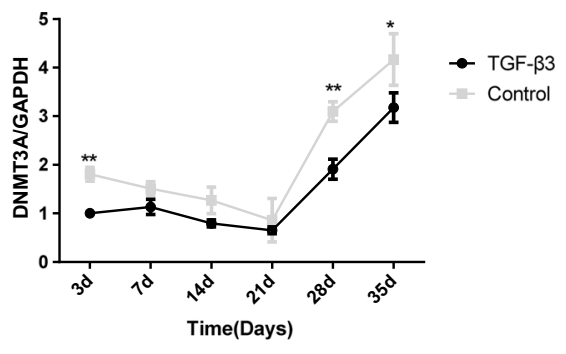

C

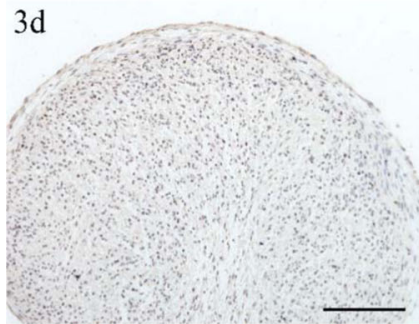

$21 \mathrm{~d}$

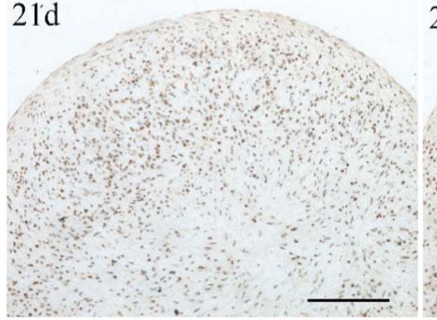

$7 \mathrm{~d}$

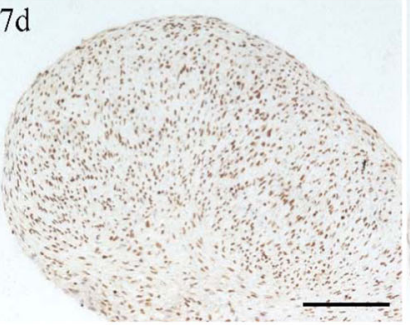

$28 \mathrm{~d}$

d

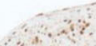

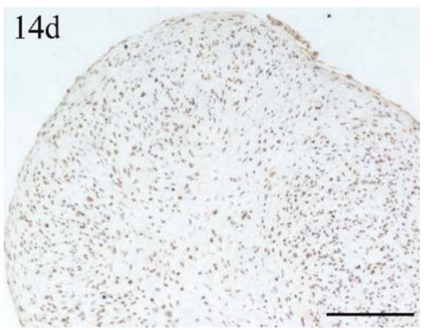

$35 \mathrm{~d}$
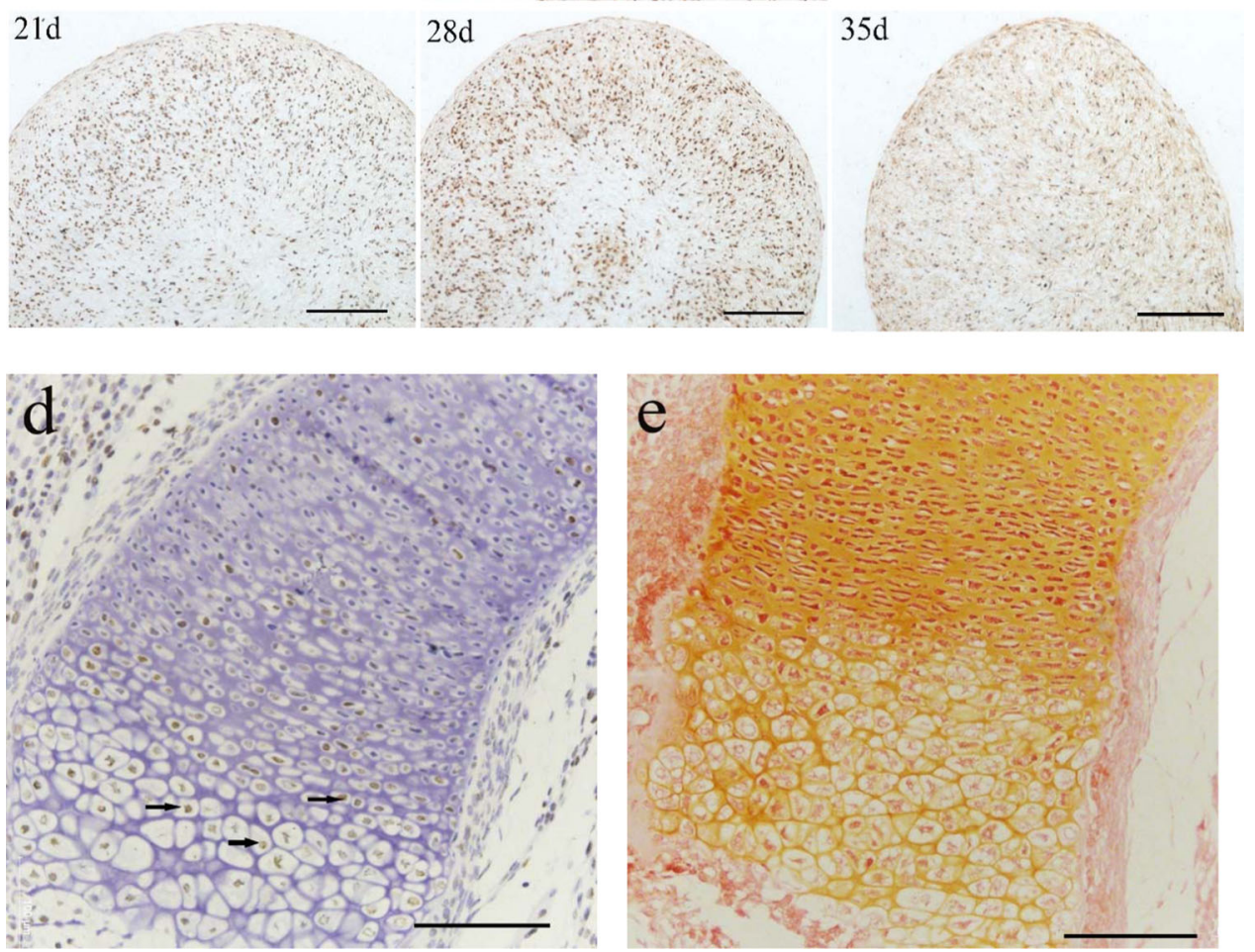

Fig. 1 Analysis of miR-455-3p and DNMT3A expression during chondrogenesis. hBMSCs cultured in chondrogenic differentiation medium for 0 , $7,14,21,28$, and 35 days. The expression of miR-455-3p (a), DNMT3A (b) were detected by qRT-PCR. hBMSCs cultured without TGF- $\beta 3$ at corresponding time points served as negative controls. U6 and GAPDH expression levels were measured and used as internal controls for microRNA and mRNA expression, respectively. hBMSCs cultured with TGF- $\beta 3$ were stained with immunohistochemistry of DNMT3A (c). The humerus in mouse embryos at E16.5 were immunostained with DNMT3A (d) and safranin O (e), scale bar, $100 \mu \mathrm{m}$. Data are presented as means \pm SD of the results obtained from triplicate samples. ${ }^{*} P<0.05 ;{ }^{*} P<0.001$ vs. negative controls, respectively 


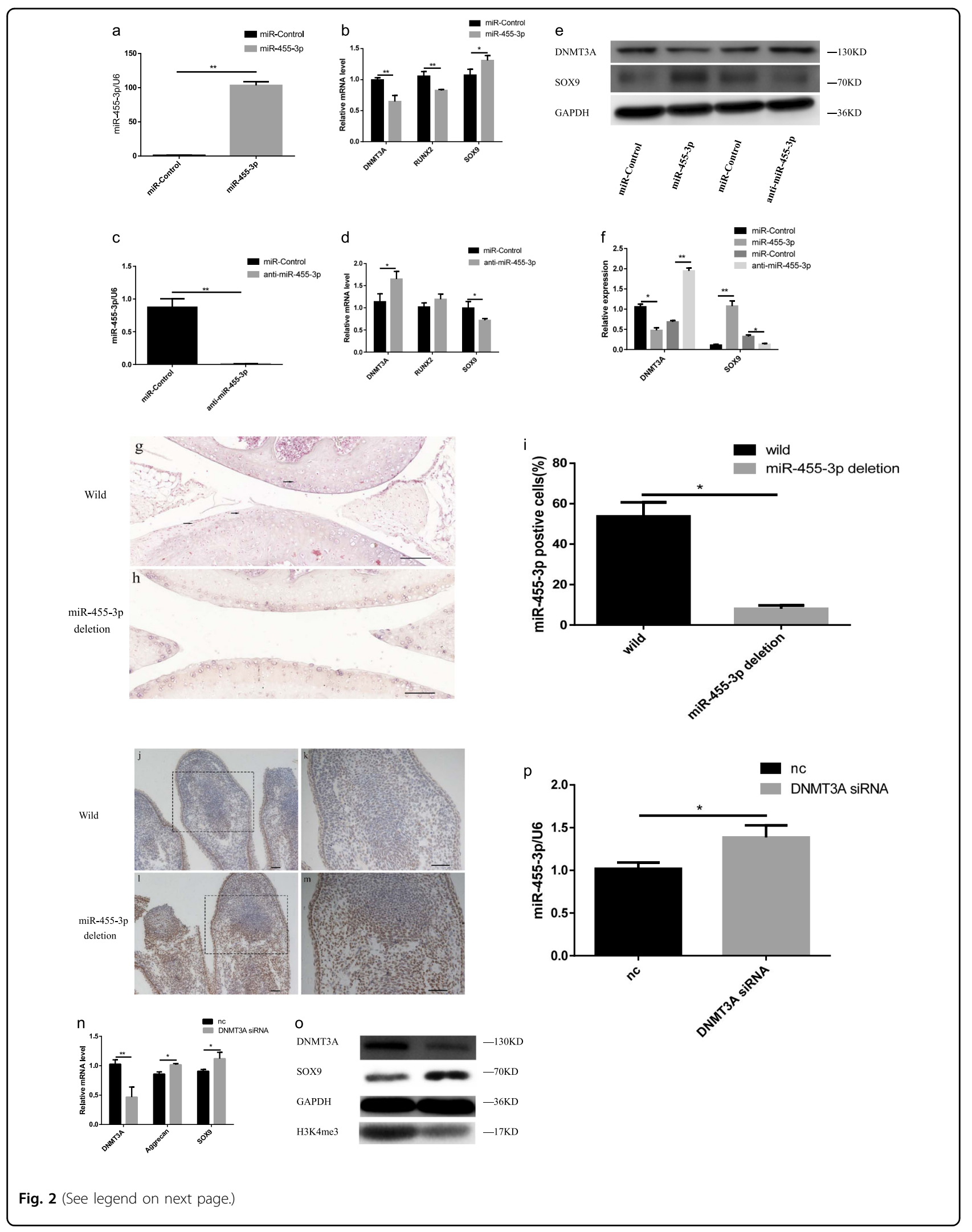


(see figure on previous page)

Fig. 2 miR-455-3p inhibits the expression of DNMT3A, and DNMT3A also modulated expression of miR-455-3p. Normal chondrocytes were transfected with miR-455-3p mimic, a non-specific control (NC) mimic, miR-455-3p inhibitor, NC inhibitor. The effect of overexpressed (a, b) or inhibited (c, d) miR-455-3p on DNMT3A, RUNX2, and SOX9 were detected by RT-PCR. Western blot analysis of DNMT3A and SOX9 expression levels $(\mathbf{e})$, and quantification (f). Situ hybridization of knee cartilage $(\mathbf{g}, \mathbf{h})$ showed the miR-455-3p expression in miR-455-3p deletion mice (h) was repressed compared with wild mice (g), scale bar, $100 \mu \mathrm{m}$. The expression of DNMT3A in palm of wild mice $\mathbf{j}, \mathbf{k})$ and miR-455-3p deletion mice (I, $\mathbf{m})$, scale bar, $100 \mu \mathrm{m}$. DNMT3A siRNA was transfected into normal chondrocytes, then RT-PCR and western blot were used to detect the expression of DNMT3A, Aggrecan, Sox9, H3K4me3 (n, o) and miR-455-3p (p). Data are presented as means \pm SD of the results of three independent experiments. ${ }^{*} P<0.05$, ${ }^{*} P<0.001$. GAPDH expression levels were measured and used as internal controls. The experiment was performed in triplicate and a representative image is shown

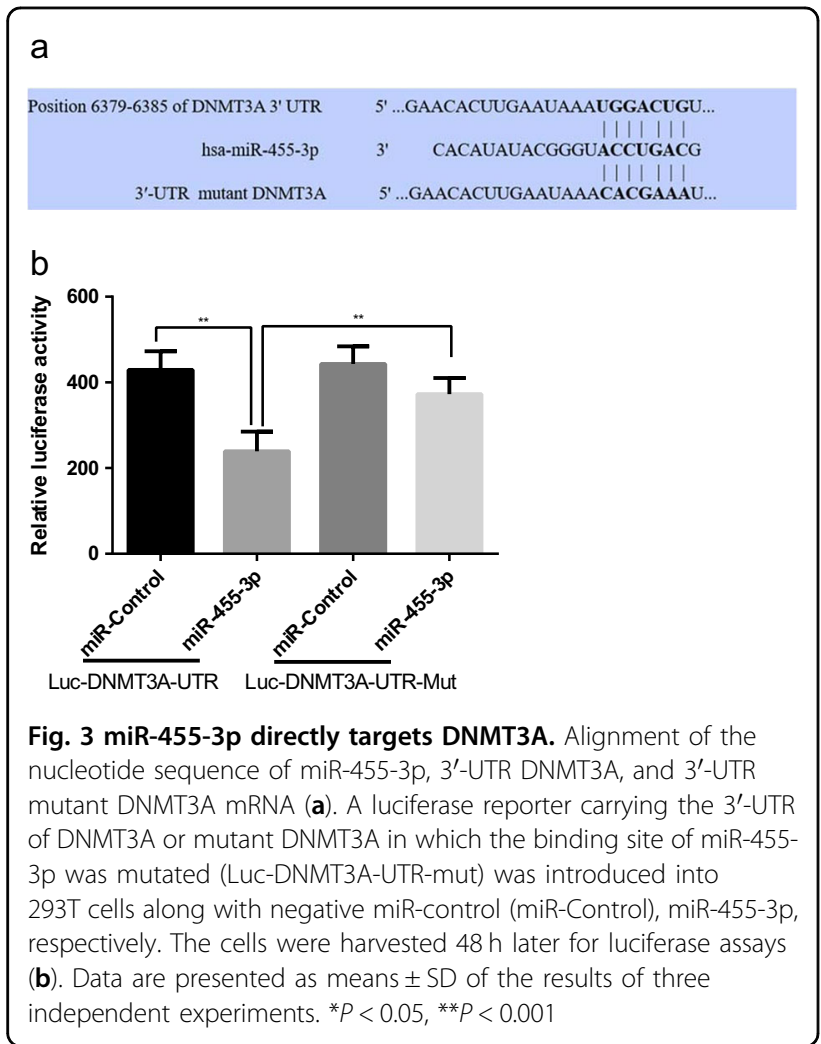

DNMT3A modulated expression of endogenous miR-455$3 p$ in normal chondrocytes

To assess whether DNMT3A modulated expression of miR-455-3p in normal chondrocytes, we transfected with DNMT3A siRNA. The expression levels of miR-455-3p were assessed by qRT-PCR, and the expression of $D N M T 3 A$ was assessed by qRT-PCR and western blotting. Treatment with $D N M T 3 A$ siRNA resulted in significantly decreased expression of DNMT3Aand H3K4me3 (Fig. 2n, o), and increased expression of miR455-3p, aggrecan, and SOX9 (Fig. 2n-p).

\section{MiR-455-3p directly targets DNMT3A by interaction with the $3^{\prime}$-UTR}

TargetScan analysis identified DNMT3A as a potential target of hsa-miR-455-3p (Fig. 3a). We, therefore, utilized a luciferase reporter assay to further examine whether the DNMT3A 3'-UTR contained a sequence capable of interacting with miR-455-3p. The potential targets of the DNMT3A 3'-UTR were mutated (Fig. 3a). Luciferase reporter assays were performed on the DNMT3A 3'-UTR and the mutated $3^{\prime}$-UTR in the presence or absence of miR-455-3p overexpression. Co-transfection of DNMT3A 3'-UTR luciferase reporter plasmids with miR-455-3p resulted in significantly decreased luciferase activity (44.3\%), and the mutation of the miR-455-3p binding sequence significantly diminished this effect (Fig. 3b). The inhibitory rate of miR-455-3p on the wild-type $3^{\prime}$-UTR DNMT3A was $44.3 \%$, while it decreased to $16.0 \%$ with the mutation of the DNMT3A 3'-UTR (Fig. 3b). Together these data indicate that miR-455-3p does modulate DNMT3A expression by binding to the $3^{\prime}$-UTR, and that $D N M T 3 A$ is a target of miR-455-3p.

\section{miR-455-3p inhibits the degenerate process during chondrogenic differentiation of hBMSCs}

Our previous results showed miR-455-3p expression rapidly increasing from day 3 to day 21 , followed by a marked decrease from days 28 to 35 during chondrogenic differentiation. Previous studies also demonstrated that miR-455-3p modulates cartilage development and degeneration ${ }^{14,15}$. Therefore, we overexpressed miR-455$3 \mathrm{p}$ in hBMSCs and induced them to differentiate into chondrocytes in micromass culture. Notably, safranin $\mathrm{O}$ staining showed that overexpression of miR-455-3p did not affect cartilage development between days 0 and 14 , but later inhibited matrix degeneration from days 21 to 35 (Fig. 4a). We then extracted the RNA on day 28 of chondrogenic differentiation to detect the expression of miR-455-3p and cartilage-related mRNAs. Treatment with the miR-455-3p agomir significantly increased the expression of miR-455-3p (Fig. 4b), COL2A1, COL11A1, and SOX6 (Fig. 4c). We also observed the cartilage thickness of hip joint in wild-type mice (Fig. 4d) and miR455-3p deletion mice at 6 months (Fig. 4e); the miR-455$3 p$ deletion mice exhibited more hypertrophic chondrocytes and thinner cartilage than wild mice (Fig. 4f). These results show that the upregulation effect of the miR-455-3p agomir in hBMSCs can continue for more 


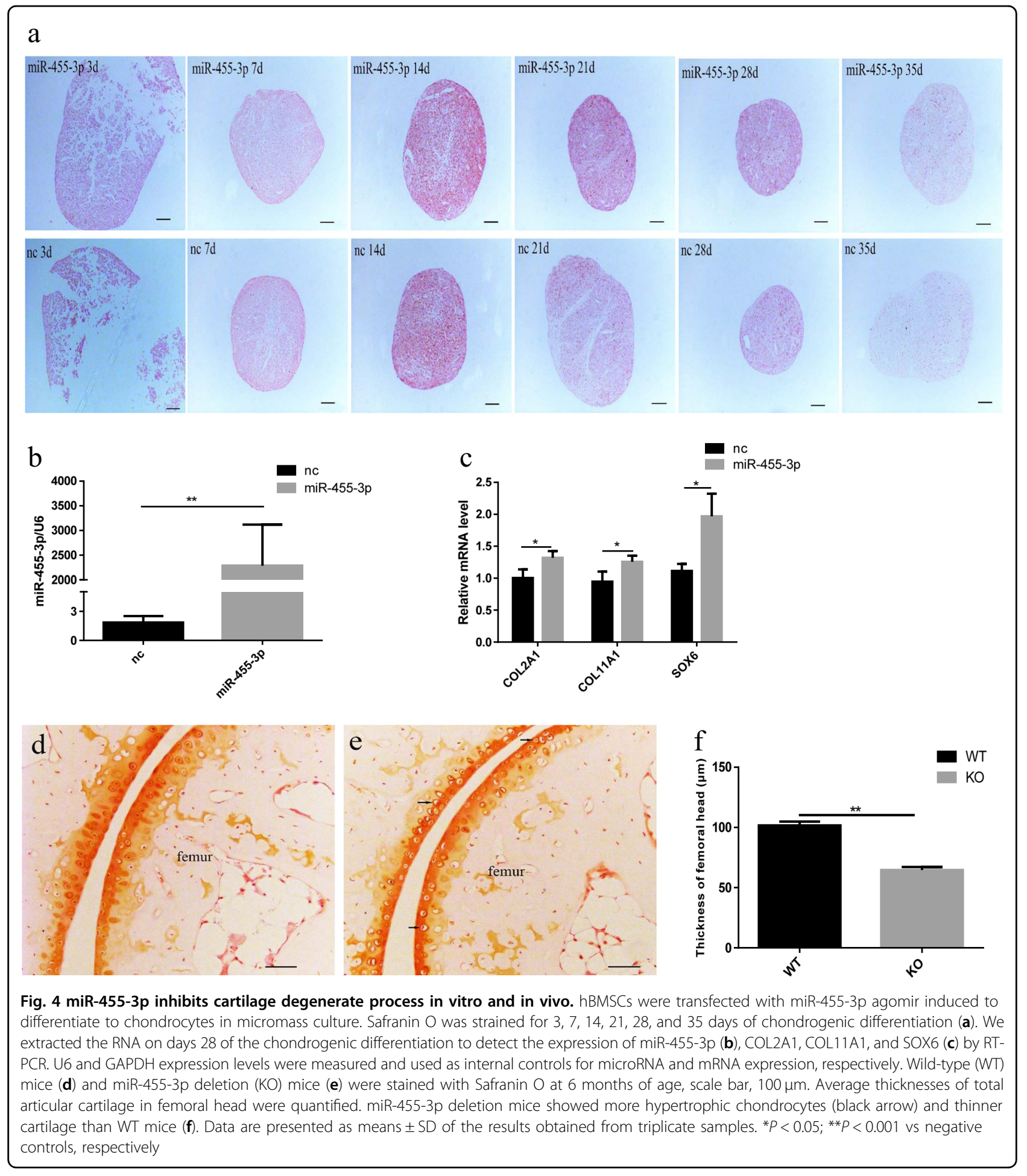

than 28 days during chondrogenic differentiation. High expression levels of miR-455-3p can inhibit degeneration of the cartilage matrix and increase the expression of cartilage-specific genes, and miR-455-3p deletion mice showed accelerated cartilage degeneration compared with wild mice.

\section{Significant differential CpG methylation between} overexpressed miR-455-3p and control groups in hBMSCs induced to differentiate to chondrocytes

To determine whether miR-455-3p regulates DNA methylation during the chondrogenic differentiation of hMSCs, genome-wide DNA methylation was examined. 
The DNA methyltransferase DNMT3A modifies mammalian genomes by cytosine methylation, an epigenetic mark that is essential for normal development and primarily occurs at CpG dinucleotides ${ }^{18}$. Most CpG-rich regions ( $\mathrm{CpG}$ islands) overlap with proximal promoters and are linked to gene silencing ${ }^{19}$. We have demonstrated that miR-455-3p inhibits the degeneration process during the chondrogenic differentiation of hBMSCs, and directly targets DNMT3A by interaction with the $3^{\prime}$-UTR. Therefore, we concluded that miR-455-3p may regulate the chondrogenic differentiation of hBMSCs by directly targeting DNMT3A to affect genomic methylation levels. As described previously, hBMSCs transfected with miR455-3p agomir or normal controls were induced to differentiate into chondrocytes in micromass culture. We compared the CpG methylation on day 28 of chondrogenic differentiation.

We detected 803,869 methylated CpG sites and observed 1733 differentially methylated sites $(P<0.01)$ representing 1023 distinct genes and nearby genomic regions when comparing miR-455-3p groups with control groups (Fig. 5a, Supplementary Figure 3a). Of these 1773 sites, 1491 (838 genes) were hypomethylated and the remaining 282 sites (183 genes) were hypermethylated (Supplementary Table 1). The top $20 \mathrm{CpGs}$ according to difference in methylation levels were listed in Table 1.

\section{Gene ontology (GO) and pathway analysis of differentially methylated CpG sites}

GO categories included biological processes, cellular components, and molecular functions, for which cellular component organization, cell junctions, and protein binding were the most diverse terms, respectively (Supplementary Figure 3b), while the PI3K-Akt signaling pathway was the most discrepant pathway (Supplementary Figure 3c). We analyzed the hypomethylation and hypermethylation sites, respectively. In the $\mathrm{GO}$ analysis, the genes related to positive regulation of NF-kB transcription factor activity were the most differentially upregulated by methylation $\left(P=1.35 \times 10^{-6}, \mathrm{FDR}=9.2 \times\right.$ $10^{-5}$ ), while the genes related to signal transduction were most differentially downregulated by methylation $(P=$ $1.39 \times 10^{-14}, \mathrm{FDR}=3.33 \times 10^{-10}$ ). The top five terms of $\mathrm{GO}$ analysis according to difference in methylation levels are listed in Table 2. Notably, the genes related to collagen biosynthesis $\left(P=1.14 \times 10^{-7}, \quad \mathrm{FDR}=3.42 \times 10^{-5}\right)$ and positive regulation of cartilage development $(P=0.005$, $\mathrm{FDR}=0.09)$ are hypomethylated. In the pathway analysis, the endocytosis pathway was the most hypermethylated $(P=0.04, \mathrm{FDR}=1)$, while the PI3K-Akt signaling pathway is the most hypomethylated $\left(P=1.20 \times 10^{-19}\right.$, FDR $\left.=1.53 \times 10^{-} 7\right)$. The ECM-receptor interaction was also hypomethylated $(P=0.002$, FDR $=0.015)$, as shown in Table 3. To confirm the DNA methylation analysis results, we overexpressed miR-455-3p in hBMSCs and induced them to differentiate to chondrocytes in micromass culture for 28 days, and immunohistochemistry of Akt and p-Akt were used to evaluate the PI3K/Akt signaling pathway. Overexpression of miR-455-3p upregulated p-Akt expression (Fig. 5b-f).

\section{Overexpression of miR-455-3p reduced the methylation of cartilage development-related genes}

Next, we analyzed the CpG methylation of cartilage development-related genes such as COL5A1, COL11A1, SMAD3, SOX6, FOXO3A, MTOR. Most of these genes are hypomethylated, except for COL13A1 and COL29A1 (Fig. 5g). Notably, most of these hypomethylated genes are related to the PI3K-Akt signaling pathway and/or the ECM-receptor interaction pathway. For example, collagen type $\mathrm{V}$ alpha 1 (COL5A1), a gene that promotes the anabolism of cartilage ${ }^{20}$, was hypomethylated with the overexpression of miR-455-3p, and is also related to the PI3K-Akt signaling pathway and the ECM-receptor interaction pathway. These data suggest that miR-455-3p inhibits the degeneration process of chondrogenic differentiation by activating canonical pathways such as the PI3K-Akt signaling pathway through the reduction of its methylation levels.

\section{Discussion}

In this study, we first identified the co-regulating activity of DNMT3A and miR-455-3p in DNA methylation during the degenerate stage of chondrogenic differentiation. Our results demonstrated that miR-455-3p regulated the expression of $D N M T 3 A$, and $D N M T 3 A$ also modulated miR-455-3p expression in vitro. The in vivo study showed high DNMT3A expression levels in miR455-3p deletion mice and low levels in wild-type mice. We also observed thinner cartilage thickness in miR-455-3p deletion mice at 6 months of age than we did in wild-type mice. Additionally, a luciferase reporter assay demonstrated that miR-455-3p regulates DNMT3A expression by binding to the DNMT3A 3'-UTR. Overexpression of miR-455-3p inhibits the degenerate process during chondrogenic differentiation by regulating DNA methylation of cartilage development-related genes and pathways.

In recent years, miRNAs have drawn increasing interest due to their regulation of a wide variety of genes, including the cartilage-specific genes ${ }^{21,22,23}$. MiR-455 is located in an intronic region of $C O L 27 A 1^{24}$, Swingler et al. reported that miR-455-3p is highly expressed during the chondrogenesis of ATDC5 cells, and that it regulates TGF $\beta$ signaling and suppresses the Smad2/3 pathway ${ }^{25}$. We also identified eight miRNAs with over twofold increases in regulation during chondrogenic differentiation of hADSCs, such as miR-320c, miR-92a-3p, and miR-455-3p ${ }^{13}$. Recent studies 


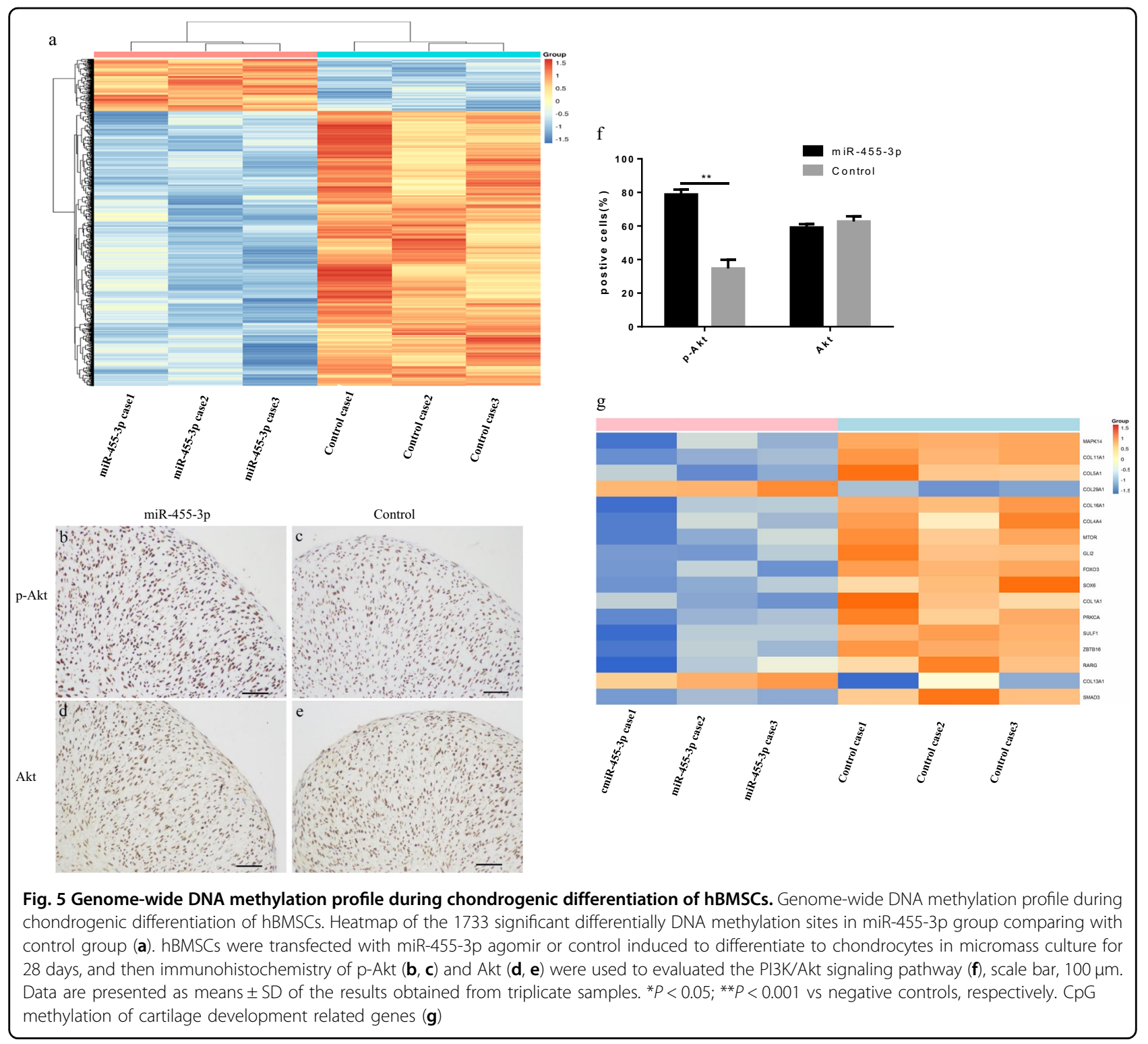

have shown that miR-455-3p functions as suppressors of hypoxia signaling ${ }^{24}$ and gastric cancer ${ }^{26}$. Our previous studies demonstrated that miR-455-3p regulated hMSC chondrogenic differentiation by targeting RUNX $2^{15}$, $H D A C 2$, and $H D A C 8^{14}$.

Moreover, our results also demonstrated that miR-455$3 \mathrm{p}$ targets the $3^{\prime}$-UTR of DNMT3A mRNA and downregulates its expression. DNMT3A is a de novo DNA methyltransferase that regulates DNA methylation, thus implicating it in diverse biological processes. MiR-101 affects lung cancer progression by targeting DNMT3A to regulate the PTEN/AKT signaling pathway ${ }^{27}$. MiR-200b$3 p$ inhibits the secretion of MMPs and promotes the synthesis of collagen type II in OA chondrocytes by inhibiting the expression of $D N M T 3 A^{28}$. In a previous study, knockdown of DNMT3A upregulated the transcription of arterial marker genes $E 2 F 1$, thereby promoting the arterial specific differentiation of hMSCs ${ }^{29}$. Our study reveals that overexpression of miR-455-3p inhibits matrix degeneration and increases the expression of the cartilage-specific genes COL2A1,COL11A1, and SOX6 during the degenerate stage of chondrogenic differentiation (days 21-35).

It has long been recognized that DNA methylation plays an important role in the occurrence and development of osteoarthritis. The differential DNA methylation of genes including $A G O 2$, TGFB3, and $H D A C 4$ were observed when comparing the cartilage intact with subchondral bone and overlying cartilage in patients with hip osteoarthritis ${ }^{30}$. Another study suggested that DNA methylation of cartilage-related genes and pathways changes in $\mathrm{OA}$ patients, especially at the late stage of $\mathrm{OA}^{31}$. Recent studies 
Table 1 Top differentially hypomethylated and hypermethylated CpG sites

\begin{tabular}{|c|c|c|c|c|c|c|c|}
\hline Illumina ID & Associated gene symbol & $\operatorname{miR}-455-3 p \beta$ & Control $\beta$ & $\Delta \beta$ & FDR-P value & CpG location & Location in enhancer \\
\hline \multicolumn{8}{|c|}{ Top hypermethylated sites } \\
\hline cg03411918 & & 0.85429915 & 0.654286759 & 0.20001239 & 0.002329516 & & NA \\
\hline cg26523866 & & 0.732835906 & 0.567929817 & 0.16490609 & 0.001328907 & & TRUE \\
\hline cg19695521 & HCG18 & 0.411955285 & 0.301119676 & 0.110835609 & 0.009617246 & Body & NA \\
\hline cg15348274 & ABCB9 & 0.290573575 & 0.18011309 & 0.110460485 & 0.001972283 & $5^{\prime}-U T R$ & NA \\
\hline cg18633134 & & 0.437928402 & 0.327541967 & 0.110386435 & 0.005910667 & & NA \\
\hline cg09916763 & NEBL & 0.689488949 & 0.584134654 & 0.105354296 & 0.000871015 & Body & NA \\
\hline cg21691809 & CLGN & 0.133046694 & 0.033722514 & 0.09932418 & 8.33E-05 & TSS200 & NA \\
\hline cg24570624 & C14orf4 & 0.331959975 & 0.234604181 & 0.097355794 & 0.00913907 & 1st Exon & NA \\
\hline cg19082105 & SLC34A1 & 0.63876831 & 0.541841261 & 0.096927048 & 0.000437297 & TSS1500 & NA \\
\hline cg06254044 & C11orf65 & 0.834485137 & 0.745398323 & 0.089086815 & 0.001994917 & TSS1500 & NA \\
\hline cg16074241 & CSMD3 & 0.712575497 & 0.62506252 & 0.087512977 & 0.000733914 & Body & NA \\
\hline cg23893009 & & 0.906343875 & 0.828571632 & 0.077772244 & 7.06E-05 & & NA \\
\hline cg11705100 & ADRA1A & 0.900905762 & 0.823951001 & 0.076954761 & 0.008305623 & Body & NA \\
\hline cg14503200 & ROR2 & 0.865238588 & 0.789787824 & 0.075450764 & 0.002193837 & Body & NA \\
\hline cg27269993 & ALB & 0.886397836 & 0.81168735 & 0.074710486 & 0.003122595 & Body & TRUE \\
\hline cg04177989 & LINC00564 & 0.433394046 & 0.360589791 & 0.072804255 & 0.004139182 & TSS1500 & NA \\
\hline cg17793819 & $\mathrm{ZHX} 2$ & 0.165543694 & 0.094208571 & 0.071335123 & 0.002716411 & TSS200 & NA \\
\hline cg20085197 & & 0.742920417 & 0.673617976 & 0.069302441 & 0.007788236 & & NA \\
\hline cg11058939 & & 0.879068917 & 0.810159701 & 0.068909215 & 0.000140968 & & TRUE \\
\hline cg12552770 & IREB2 & 0.932863243 & 0.867463255 & 0.065399989 & 0.003824771 & Body & NA \\
\hline \multicolumn{8}{|c|}{ Top hypomethylated sites } \\
\hline cg00449608 & GPR45 & 0.242981495 & 0.686573289 & -0.443591794 & 0.009516558 & TSS1500 & NA \\
\hline cg06198776 & PIBF1 & 0.154669829 & 0.572247756 & -0.417577928 & $3.35 \mathrm{E}-05$ & Body & NA \\
\hline cg19450830 & LINC00548 & 0.39194816 & 0.7977394 & -0.40579124 & 0.000220008 & TSS1500 & TRUE \\
\hline cg07374695 & $\mathrm{P} 2 \mathrm{RX} 7$ & 0.28044041 & 0.682154337 & -0.401713928 & 0.001304077 & Body & NA \\
\hline cg09627772 & RABGAP1L & 0.162932352 & 0.561519876 & -0.398587524 & 0.000884627 & Body & NA \\
\hline cg05007112 & GCC2 & 0.171615788 & 0.563873804 & -0.392258017 & 0.00172162 & Body & NA \\
\hline cg14708360 & & 0.188967691 & 0.572140844 & -0.383173152 & 0.000467635 & & TRUE \\
\hline cg07475284 & HECW2 & 0.419477422 & 0.794862044 & -0.375384622 & 0.00024201 & $5^{\prime}-U T R$ & TRUE \\
\hline cg22130673 & ZBTB16 & 0.37642215 & 0.748233855 & -0.371811705 & 0.001092287 & Body & TRUE \\
\hline cg00608183 & & 0.227348067 & 0.598587288 & -0.371239221 & 0.000506866 & & NA \\
\hline cg00979515 & & 0.222629852 & 0.593228804 & -0.370598952 & 7.97E-05 & & NA \\
\hline cg02390981 & $\mathrm{KLC1}$ & 0.408629455 & 0.77894639 & -0.370316935 & 0.000404981 & Body & NA \\
\hline cg22602094 & ADAMTS16 & 0.163089519 & 0.524240086 & -0.361150567 & 0.004151807 & Body & NA \\
\hline cg13201036 & EPN2 & 0.095664948 & 0.454884995 & -0.359220047 & 0.000512488 & Body & NA \\
\hline cg11769597 & & 0.145825087 & 0.502098007 & -0.35627292 & 0.000452804 & & NA \\
\hline c g05559888 & & 0.254035348 & 0.608152643 & -0.354117295 & 0.003276138 & & NA \\
\hline cg20224091 & & 0.11584416 & 0.469822353 & -0.353978192 & $1.35 \mathrm{E}-05$ & & NA \\
\hline
\end{tabular}


Table 1 continued

\begin{tabular}{cllllllll}
\hline Illumina ID & Associated gene symbol & miR-455-3p $\boldsymbol{\beta}$ & Control $\boldsymbol{\beta}$ & $\boldsymbol{\Delta} \boldsymbol{\beta}$ & FDR-P value & CpG location & Location in enhancer \\
\hline cg21542303 & NFKB1 & 0.188709635 & 0.541788057 & -0.353078422 & 0.002441491 & Body & NA \\
cg23295942 & & 0.228562019 & 0.580751528 & -0.352189509 & 0.005733339 & & NA \\
cg13280925 & SEC63 & 0.411386516 & 0.760081635 & -0.348695119 & 0.002720771 & Body & NA \\
\hline
\end{tabular}

$\Delta \beta$ difference in methylation value between sample groups $\left(\beta_{\text {miR-455-3p }}-\beta_{\text {control }}\right)$, FDR false discovery rate, NA not applicable, TSS 1500 within 1500 bp of transcription start site, 5'-UTR 5'-untranslated region.

Table 2 Gene ontology analysis

\begin{tabular}{|c|c|c|c|}
\hline Pathway & No. of DM genes & $P$ & FDR \\
\hline \multicolumn{4}{|l|}{ Hyper-methylated } \\
\hline Positive regulation of NF-kB transcription factor activity & 7 & $1.35 \mathrm{E}-06$ & 0.000920046 \\
\hline Transmembrane transport & 12 & $9.41 \mathrm{E}-06$ & 0.003198452 \\
\hline Embryonic skeletal system development & 4 & $1.99 \mathrm{E}-05$ & 0.004507739 \\
\hline Anterior/posterior pattern specification & 5 & 0.000131002 & 0.022270268 \\
\hline Multicellular organismal homeostasis & 2 & 0.000195932 & 0.026646776 \\
\hline \multicolumn{4}{|l|}{ Hypo-methylated } \\
\hline Signal transduction & 63 & $1.39 \mathrm{E}-13$ & $3.33 \mathrm{E}-10$ \\
\hline Positive regulation of transcription from RNA polymerase II promoter & 46 & $5.75 E-11$ & $6.87 \mathrm{E}-08$ \\
\hline Small molecule metabolic process & 67 & $4.21 \mathrm{E}-10$ & 3.35E-07 \\
\hline Negative regulation of transcription from RNA polymerase II promoter & 36 & $9.78 \mathrm{E}-10$ & $5.85 \mathrm{E}-07$ \\
\hline Intracellular signal transduction & 25 & 6.94E-09 & $3.32 \mathrm{E}-06$ \\
\hline
\end{tabular}

DM differentially methylated

Table 3 Differentially methylated canonical pathways between miR-455-3p and control groups

\begin{tabular}{llll}
\hline Pathway & $\begin{array}{l}\text { No. of DM } \\
\text { genes }\end{array}$ & $\boldsymbol{P}$ & FDR \\
\hline $\begin{array}{l}\text { Hyper-methylated } \\
\quad \text { Endocytosis }\end{array}$ & 4 & 0.039186726 & 1 \\
Hypo-methylated & & & \\
Pl3K-Akt signaling pathway & 29 & $1.20652 \mathrm{E}-09$ & $1.53573 \mathrm{E}-07$ \\
Pathways in cancer & 28 & $1.40249 \mathrm{E}-09$ & $1.53573 \mathrm{E}-07$ \\
Metabolic pathways & 47 & 0.000117961 & 0.001987193 \\
Wnt signaling pathway & 11 & 0.000824249 & 0.009500549 \\
ECM-receptor interaction & 8 & 0.001821074 & 0.015339048 \\
\hline
\end{tabular}

have shown that DNA methylation has emerged as a crucial regulator of chondrocyte dedifferentiation. Treatment with the DNA methylation inhibitor 5-azacytidine reverses the chondrocyte dedifferentiation and increases SOX-9 expression $^{32}$. Our study demonstrated that miR-455-3p plays a role similar to 5-azacytidine in repressing DNMT3A expression and inhibiting DNA methylation. miR-455-3p overexpression downregulates the DNA methylation of cartilage development-related genes such as SOX6 and Smad3. Both upregulation of $S O X 6^{33}$ and $S m a d 3^{34}$ promotes chondrogenic differentiation of hMSCs.

The PI3K-Akt signaling pathway is essential for the process of differentiation ${ }^{35}$. Activation of the pathway enhance chondrocyte proliferation and inhibit hypertrophic differentiation ${ }^{36,37}$, and its inhibition increases apoptosis $^{38}$. GO analysis of differentially methylated sites revealed the PI3K-Akt signaling pathway as the most hypomethylated pathway. These data demonstrate that miR-455-3p can inhibit the expression of DNMT3A to regulate DNA methylation of cartilage-specific genes, thus regulating pathways such as the PI3K-Akt signaling pathway and ultimately inhibiting the cartilage degeneration during chondrogenic differentiation.

In summary, this study demonstrates that miR-455-3p and DNMT3A co-regulate chondrogenic differentiation of hMSCs by modulating DNA methylation. The increased expression of miR-455-3p and decreased expression of DNMT3A upregulate cartilage-specific genes and 
pathways, thereby attenuating cartilage degeneration during chondrogenic differentiation. To the best of our knowledge, this study is the first to demonstrate the coregulation between DNMT3A and miR-455-3p in vivo and the chondrogenic differentiation of hBMSCs in vitro. These results not only reveal the important role of DNA methylation in chondrogenic differentiation, but also demonstrate that miR-455-3p can regulate this process by affecting DNA methylation. Overexpression of miR-455$3 p$ and the use of a DNA methylation inhibitor could optimize the process of chondrogenic differentiation.

\section{Materials and methods}

All procedures were approved by the ethical committee of the First Affiliated Hospital of Sun Yat-Sen University (IRB: 2014C-028) and the Helsinki Declaration (2000). All volunteers provided written informed consent.

\section{Articular chondrocyte isolation and culture}

Human normal articular cartilage samples were obtained from five patients (one male and four females; mean \pm SD age, $65.4 \pm 4.0$ years) who had no history of OA or rheumatoid arthritis and who underwent hemiarthroplasty or total hip arthroplasty because of femoral neck fractures. Informed consent was obtained from all patients. The cartilages were dissected away from the subchondral bone and then digested by $4 \mathrm{mg} / \mathrm{mL}$ protease and $0.25 \mathrm{mg} / \mathrm{mL}$ collagenase $\mathrm{P}$ as described previously ${ }^{16}$. Cells were cultured in DMEM/Nutrient Mixture F-12 (Gibco Life Technology Grand Island, NY, USA) containing 5\% fetal bovine serum (FBS; Gibco Life Technology), $100 \mathrm{IU} / \mathrm{mL}$ penicillin, and $100 \mu \mathrm{g} / \mathrm{mL}$ streptomycin (Gibco Life Technology). The chondrocytes were used in experiments within 3-7 days and without passaging to avoid dedifferentiation.

\section{Human mesenchymal stem cell isolation, culture, and chondrogenic differentiation}

Bone marrow samples were obtained from three normal human donors (one male and two females; mean \pm SD age, $25.3 \pm 3.8$ years), and hBMSCs were isolated as described previously ${ }^{16}$. Cells were cultured in Alphamodified Eagle's medium (a-MEM) (Gibco Life Technology Grand Island, NY, USA) supplemented with $10 \%$ FBS, $100 \mathrm{IU} / \mathrm{mL}$ penicillin, and $100 \mu \mathrm{g} / \mathrm{mL}$ streptomycin. The hMSCs were induced to differentiate into chondrocytes in micromass culture within three passages as described previously ${ }^{16}$. Briefly, hBMSCs were resuspended in aMEM supplemented with $10 \%$ FBS at a specified density of $10^{5}$ cells $/ \mu \mathrm{L}$ of media, and $12.5 \mu \mathrm{L}$ of the suspended cells were dotted on the center of each well of 24-well plates and then cultured in the $37^{\circ} \mathrm{C}$ incubator for $1 \mathrm{~h}$ to stimulate the adherence of the cells to the plate. Next, the cells were cultured with chondrogenic induction medium (human mesenchymal stem cell chondrogenic differentiation basal medium $97 \mathrm{~mL}$, TGF- $\beta 31 \mathrm{~mL}$, dexamethasone $10 \mu \mathrm{L}$, ascorbate $300 \mu \mathrm{L}$, ITS (insulin, transferrin, selenium) supplement $1 \mathrm{~mL}$, sodium pyruvate $100 \mu \mathrm{L}$, proline $100 \mu \mathrm{L}$ ) (Cyagen, Guangzhou, China), or incomplete chondrogenic induction medium without TGF- $\beta 3$ as control. At indicated time points, the cells were subjected to RNA extraction or stained with safranin $\mathrm{O}$ or immunohistochemistry.

\section{Animals and in situ hybridization}

The mmu-miR-455-3p global knockout mice were generated by a transcription activator-like effector nuclease (TALEN) system. Briefly, we use $1 \mu \mathrm{m}$ of linearized plasmid as a template for an in vitro transcription reaction. Next, synthesized RNA was treated with Poly(A) Tailing Kit (Life Technologies) to add poly(A) tail. Microinjection of a mixture of RNAs into cytoplasm of one-cell stage embryo, and then two-cell stage embryos were transferred into pseudopregnant C57BL/6 female mice. For genotyping, DNA sequencing analysis was performed after PCR. The wild-type (miR-455-3p $+/+$ ), heterozygous (miR-455-3p $+/-$ ), and homozygous (miR-455-3p-l-) mice were obtained through the mating of heterozygous male and female mice. In our study, we compared the wild-type mice and homozygous mice.

The knee joint of miR-455-3p deletion mice and wildtype mice were harvested at 6 months and fixed by incubation in DEPC-treated $10 \%$ formalin at $4{ }^{\circ} \mathrm{C}$ overnight. Tissues were subsequently dehydrated with a graded series of ethanol, embedded in paraffin, and cut into 5 - $\mu$ m-thick sections. Sections were subjected to in situ hybridization analysis using the miR- 455-3p-specific probe (Servicebio, Wuhan, China), or treated with PBS instead of the miR-455-3p probe as negative control.

\section{Transfection}

The normal chondrocytes were transfected with miR455-3p mimic or inhibitor (RiboBio, Guangzhou, China) at a concentration of $50 \mathrm{nM}$; they were also transfected with DNMT3A-siRNA or NC (RiboBio, Guangzhou, China). Lipofectamine 2000 Transfection Reagent (Gibco Life Technologies) was used to transfect cells according to the manufacturer's instructions. Cells were then harvested after $48 \mathrm{~h}$ for quantitative real-time reverse transcriptionpolymerase chain reaction (qRT-PCR), or after $72 \mathrm{~h}$ for western blot analysis.

\section{RT-PCR analysis, western blot, and immunohistochemistry}

Total cellular RNA was isolated using miRNeasy Mini Kit (Qiagen, Venlo, Netherlands). Next, cDNA was synthesized from mRNA and miRNA by using PrimeScript ${ }^{\text {mix }}$ RT Master Mix (Takara, Shiga, Japan) and Mir- $\mathrm{X}^{\mathrm{m}}$ 
miRNA First-Strand Synthesis Kit (Clontech Laboratories, Inc., Mountain View, CA, USA), respectively. qPCR of miR-455-3p and target mRNAs were performed using SYBR $^{\circ}$ Premix Ex Taq $^{\text {Ta }}$ II (Takara) and KOD SYBR ${ }^{\circ}$ qPCR Mix(Toyobo), according to the manufacturer's instructions, respectively. Transcript levels were normalized to that of the housekeeping gene glyceraldehyde 3phosphate dehydrogenase (GAPDH; for mRNA) or the small U6 RNA (for miRNA). The specific primers used for these analyses are listed in Supplementary Table 2. Gene expression was calculated using the $2^{-\Delta \Delta \mathrm{Ct}}$ method, and each experiment was performed in triplicate. Western blotting analysis was performed as described previously ${ }^{16}$. Briefly, total proteins were isolated from normal or OA chondrocytes by RIPA buffer (Beyotime Biotechnology, Beijing, China) containing protease inhibitors (Abcam) to obtain whole cell extracts. Membranes were incubated with primary antibodies (1:1000 dilution) against DNMT3A (D23G1, Cell Signaling Technology), SOX9 (D8G8H, Cell Signaling Technology), and H3K4me3 (C42D8, Cell Signaling Technology). GAPDH (EPR16891, Abcam) was utilized as an internal control. Immunohistochemical analysis was carried out as described previously $^{16}$. Sections were treated with $3 \%$ normal goat serum for $1 \mathrm{~h}$ and incubated with antibodies specific to DNMT3A (D23G1, Cell Signaling Technology), p-Akt (D9E, Cell Signaling Technology), and Akt(C67E7, Cell Signaling Technology). Sections were also stained with safranin $\mathrm{O}$.

\section{Luciferase constructs and reporter assay}

The DNMT3A sequences of DNMT3A 3'-UTR were amplified by polymerase chain reaction (PCR) using the following primers: forward 5'-ATAGGCCGGCATAGAC GCGTAGCGGT CTAAAGCATCCC-3' and reverse 3'-A AAGATCCTTTATTAAGCTTTTATTACGGTGC TTT TCCATTT -5 . The seed sequences were mutated with the following primers: forward 5'-TTATTACGGTGCTTT TCCATTTTTCTTTTTGCACAAAACTTTCGTGTTTA TTCAAG TGTTCTCCAGCA-3' and reverse $3^{\prime}$-ACAC CGTCGAACAATCACTCTTGCATT GATTTC-5'. The amplified DNA sequences were inserted into the pmiRRB-REPORT $^{\mathrm{mm}}$ Vector (OBIO, Shanghai, China) to generate DNMT3A 3'-UTR or mutated DNMT3A 3'-UTR luciferase vectors. For the dual luciferase assay, $1.2 \times 10^{4}$ cells (HEK293T) in a 96-well plate were transfected with $50 \mathrm{nM}$ hsa-miR-455-3p or mimic NC (RiboBio). The cells were then co-transfected with $2 \mu \mathrm{g} / \mathrm{mL}$ of vector with the wild-type or mutant DNMT3A 3'-UTR. Luciferase activity was measured $48 \mathrm{~h}$ after transfection by the DualLuciferase Reporter Assay System (Promega, Madison, WI, USA) according to the manufacturer's instructions. Luciferase assays were performed in three independent experiments.

\section{Differential methylation analysis and gene ontology analyses}

Genomic DNA was extracted using QIAamp DNA Mini Kit (QIAGEN, Tokyo, Japan). After the quality and quantity identification by agarose gel electrophoresis Spectrophotometer (SpectraMax Plate Reader, Molecular Devices, Sunnyvale, CA), DNA $(1 \mu \mathrm{g})$ was bisulfite treated using an EZ DNA methylation kit (Zymo Research, Irvine, $\mathrm{CA})$, and genome-wide DNA methylation was assessed using an Illumina Human MethylationEPIC microarray (Illumina, San Diego, CA, USA), which analyzes the methylation status of $>850,000$ methylation sites throughout the genome. GenomeStudio Methylation Module v1.8 (Illumina) was used to analyze the differential methylation sites, according to the manufacturer's instructions. The percentage of methylated cytosine at each CpG site was calculated as the ratio of methylated probe signal to total locus signal intensity $(\beta: 0-1)$ by GenomeStudio, and then average $\beta$ values were compared. We defined differentially methylated CpG sites as having a $P$ value less than or equal to 0.01 after adjusting for multiple testing using GenomeStudio. The GO Database was used for $\mathrm{GO}$ analysis. Total genes were treated as the background genes, a list of differential genes was selected, and then a hypergeometric distribution calculation was used to test whether the genes in the list were significantly enriched.

\section{Statistical analysis}

Statistical analyses were performed using SPSS 19.0 software (IBM Corporation, Armonk, NY, USA). Data are presented as mean \pm standard deviation (SD) of the results of at least three independent experiments. Student's $t$-test was used to identify differences between groups. One-way analysis of variance and Kruskal-Wallis tests were used for performing multiple group comparisons. $P<0.05$ was considered statistically significant for all the tests.

\begin{abstract}
Acknowledgements
This study was funded by the National Natural Science Foundation of China (no. 81472101, 81672145, and 81572119), the Science and Technology Project of Guangdong Province, China (2014A020211003 and 2015A020212015) and the Science and Technology Project of Guangzhou City, China (201710010164). We thank Aaron Weiner from University of Pennsylvania for English revision.
\end{abstract}

\section{Conflict of interest}

The authors declare that they have no conflict of interest.

\section{Publisher's note}

Springer Nature remains neutral with regard to jurisdictional claims in published maps and institutional affiliations.

Supplementary Information accompanies this paper at (https://doi.org/ 10.1038/s41419-018-0565-2). 
Received: 1 November 2017 Revised: 19 March 2018 Accepted: 5 April 2018 Published online: 10 May 2018

\section{References}

1. Senner, C. E. The role of DNA methylation in mammalian development Reprod. Biomed. Online 22, 529-535 (2011).

2. Pellacani, D. et al. DNA hypermethylation in prostate cancer is a consequence of aberrant epithelial differentiation and hyperproliferation. Cell Death Differ. 21, 761-773 (2014).

3. Gatto, S. et al. ICF specific DNMT3B dysfunction interferes with intragenic regulation of mrna transcription and alternative splicing. Nucleic Acids Res. 45, 5739-5756 (2017)

4. Hata, K., Okano, M., Lei, H. \& Li, E. DNMT3L cooperates with the DNMT3 family of de novo DNA methyltransferases to establish maternal imprints in mice. Development 129, 1983-1993 (2002).

5. Simon, T. C. \& Jeffries, M. A. The epigenomic landscape in osteoarthritis. Curr. Rheumatol. Rep. 19, 30 (2017).

6. Andrés, M. C. D. et al. Loss of methylation in cpg sites in the NF-kB enhancer elements of inducible nitric oxide synthase is responsible for gene induction in human articular chondrocytes. Arthritis Rheum. 65, 732-742 (2013).

7. Hashimoto, K. et al. Regulated transcription of human matrix metalloproteinase 13 and interleukin-1 genes in chondrocytes depends on methylation of specific proximal promoter CpG sites. J. Biol. Chem. 288, 10061-10072 (2013).

8. Yatabe, T. et al. Hyaluronan inhibits expression of adamts4 in human osteoarthritic chondrocytes. Ann. Rheum. Dis. 68, 1051-1058 (2009).

9. Kim, K. I., Park, Y. S. \& Im, G. I. Changes in the epigenetic status of the SOX9 promoter in human osteoarthritic cartilage. J. Bone Mineral. Res. 28, 1050-1060 (2013).

10. Del Real, A. et al. Differential analysis of genome-wide methylation and gene expression in mesenchymal stem cells of patients with fractures and osteoarthritis. Epigenetics 12, 113-122 (2016).

11. Kornicka, K., Marycz, K., Marędziak, M., Tomaszewski, K. A. \& Nicpoń, J. The effects of the DNA methyltranfserases inhibitor 5-azacitidine on ageing, oxidative stress and DNA methylation of adipose derived stem cells. J. Cell. Mol. Med. 21, 387-401 (2017).

12. Bartel, D. P. MicroRNAs: genomics, biogenesis, mechanism, and function. Cell 116, 281-297 (2004).

13. Zhang, Z. et al. Expression of micrornas during chondrogenesis of human adipose-derived stem cells. Osteoarthr. Cartil. 20, 1638-1646 (2012).

14. Chen, W. et al. Microrna-455-3p modulates cartilage development and degeneration through modification of histone $\mathrm{H} 3$ acetylation. Biochim. Biophys. Acta 1863, 2881-2891 (2016)

15. Zhang, Z. et al. miR-455-3p regulates early chondrogenic differentiation via inhibiting RUNX2. FEBS Lett. 589, 3671-3678 (2015).

16. Mao, G. et al. MicroRNA-92a-3p regulates the expression of cartilage-specific genes by directly targeting histone deacetylase 2 in chondrogenesis and degradation. Osteoarthr. Cartil. 25, 521-532 (2017).

17. Zhao, Y. et al. Microrna-455-3p functions as a tumor suppressor by targeting eif4e in prostate cancer. Oncol. Rep. 37, 2449-2458 (2017).

18. Jaenisch, R. \& Bird, A. Epigenetic regulation of gene expression: how the genome integrates intrinsic and environmental signals. Nat. Genet. 33, 245-254 (2003)

19. Suzuki, M. M. \& Bird, A. DNA methylation landscapes: provocative insights from epigenomics. Nat. Rev. Genet. 9, 465-476 (2008).
20. Wang, W. et al. Comparative analysis of gene expression profiles of hip articular cartilage between non-traumatic necrosis and osteoarthritis. Gene 591, 43-47 (2016).

21. Chen, W. et al. Microrna-381 regulates chondrocyte hypertrophy by inhibiting histone deacetylase 4 expression. Int. J. Mol. Sci. 17, 1377 (2016).

22. Budd, E., Andrés, M. C. D., Sanchezelsner, T. \& Oreffo, R. O. C. MiR-146b is downregulated during the chondrogenic differentiation of human bone marrow derived skeletal stem cells and up-regulated in osteoarthritis. Sci. Rep. 7, 46704 (2017).

23. Gantier, M. P. et al. A miR-19 regulon that controls nf-kb signaling. Nucleic Acids Res. 40, 8048-8058 (2012).

24. Lalevee, S., Lapaire, O. \& Bühler, M. miR455 is linked to hypoxia signaling and is deregulated in preeclampsia. Cell Death Dis. 5, e1408 (2014).

25. Swingler, T. E. et al. The expression and function of microRNAs in chondrogenesis and osteoarthritis. Arthritis Rheum. 64, 1909-1919 (2002).

26. Ning, $T$. et al. miR-455 inhibits cell proliferation and migration via negative regulation of EGFR in human gastric cancer. Oncol. Rep. 38, 175-182 (2017).

27. Wang, L. et al. Microrna-101 suppresses progression of lung cancer through the PTEN/AKT signaling pathway by targeting DNA methyltransferase 3a. Oncol. Lett. 13, 329-338 (2017).

28. Wu, J. et al. Effect of the interaction between miR-200b-3p and DNMT3A on cartilage cells of osteoarthritis patients. J. Cell. Mol. Med. 21, 2308-2316 (2017).

29. Zhang, R. et al. Knockdown of dnmt1 and dnmt3a promotes the angiogenesis of human mesenchymal stem cells leading to arterial specific differentiation. Stem Cells 34, 1273-1283 (2016).

30. Jeffries, M. A. et al. Genome-wide DNA methylation study identifies significant epigenomic changes in osteoarthritic subchondral bone and similarity to overlying cartilage. Arthritis Rheumatol. 68, 1403-1414 (2016).

31. Zhang, Y. et al. Genome-wide DNA methylation profile implicates potentia cartilage regeneration at the late stage of knee osteoarthritis. Osteoarthr. Cartil. 24, 835-843 (2016).

32. Duan, L. et al. DNA methylation profiling in chondrocyte dedifferentiation in vitro. J. Cell. Physiol. 232, 1708-1716 (2016).

33. Wang, H., Shan, X. B. \& Qiao, Y. J. PDK2 promotes chondrogenic differentiation of mesenchymal stem cells by upregulation of Sox6 and activation of JNK MAPK/ERK pathway. Braz. J. Med. Biol. Res. 50, e5988 (2017).

34. de Kroon, L. M. et al. Smad3 and smad4 have a more dominant role than smad2 in TGF $\beta$-induced chondrogenic differentiation of bone marrowderived mesenchymal stem cells. Sci. Rep. 7, 43164 (2017).

35. Li, H. et al. MiR-216a rescues dexamethasone suppression of osteogenesis, promotes osteoblast differentiation and enhances bone formation, by regulating c-cbl-mediated PI3K-AKT pathway. Cell Death Differ. 22, 1935-1945 (2015).

36. Kita, K., Kimura, T., Nakamura, N., Yoshikawa, H. \& Nakano, T. PI3K/Akt signaling as a key regulatory pathway for chondrocyte terminal differentiation. Genes Cells 13, 839-850 (2008).

37. Li, Z., Lu, H. \& Li, Z. B. The PI3K/Akt signalling pathway may play an internal role related to abnormal condylar growth: a preliminary study. Int. J. Oral. Maxillofac. Surg. 43, 1477-1483 (2014).

38. He, B. et al. Protection of carboxymethylated chitosan on chondrocytes from nitric oxide-induced apoptosis by regulating PI3K-AKT signaling pathway. Biochem. Biophys. Res. Commun. 479, 380-386 (2016). 